\title{
Le corps du chercheur et la question esthétique dans la science géographique
}

\section{The researcher's body and the issue of aesthetics in geography}

- Anne Volvey, Professeure de géographie à l'Université d'Artois, Membre de l'EA 2468, Discontinuités. anne.volvey@ens-lyon.org-

\section{Résumé :}

Articulant les textes de la géographie féministe et qualitative anglophone des années 19902000 qui ont refondé le projet scientifique de la géographie sur un double mouvement de déconstruction/reconstruction des méthodologies de terrain, cet article développe une perspective théorique et conceptuelle sur la question de l'esthétique du savoir géographique. A partir d'une réflexion sur les motifs identitaires et les formes de corporalité caractérisant l'engagement du sujet-cherchant sur le terrain, en mobilisant un corpus de textes issus de la psychanalyse contemporaine, il construit la généalogie critique des esthétiques du savoir spatial (scopique, linguistique, haptique), avant de les rassembler dans une perspective transitionnelle.

\section{Summary:}

Putting together texts from the feminist and qualitative geographies of the 1990s and 2000s, which ones have founded the scientific project of geography on a double movement of deconstruction and reconstruction of the fieldwork methodologies, this paper aims at developing an altogether theoretical and conceptual perspective on the issue of the geographical knowledge's aesthetics. Built on a reflection about the identity motives and the forms of embodiment that characterize the fieldworker's performance, resorting to a body of texts of the contemporary psychoanalysis, this paper offers a critical perspective on the genealogy of the aesthetics of spatial knowledge (scopic, linguistic and haptic), before it articulates them within a transitional framework.

Mots clés : corps, terrain, épistémologie, esthétique, régime de savoir géographique, hapticité. Key words : body, fieldwork, epistemology, aesthetics, regime of geographical knowledge, hapticity

De nombreux auteur-e-s constatent l'avènement du corps dans les pratiques contemporaines et leur médiatisation et il est aujourd'hui un sujet d'une approche géographique informée des théories phénoménologiques ou de la pratique, qui se propose d'articuler les rapports entre pratique/expérience, corporalité/corporéité et spatialité. Sur un plan épistémologique, après un siècle d'empirisme positiviste qui l'a caché et au-delà lui a dénié un statut scientifique, l'effet retour du principe de symétrie ${ }^{1}$ associé au constructivisme et au post-modernisme place aujourd'hui le corps du/de la chercheur-e au cœur des réflexions

\footnotetext{
${ }^{1}$ Suivant Latour et Woolgar (1996), le principe de symétrie revient à appliquer à l'activité de recherche des géographes les mêmes questionnements, outils théoriques et méthodologiques que ceux qu'ils mobilisent pour travailler sur les activités de leurs sujets de recherche, afin de faire surgir comme une question épistémologique le problème du sujet-cherchant-avec-l'espace.
} 
portant sur le procès de la recherche contemporaine et au-delà sur le savoir géographique. Le corps du sujet-cherchant engagé sur le terrain de la fabrique de la science, où il est à la fois opérateur de l'activité de recherche (motricité), médiateur de la relation de recherche (émotionalité, affectivité, idéalité) et récepteur et processeur des données tirées de l'expérience empirique (sensorialité, kinesthésie, empathie) est aujourd'hui un champ de l'épistémologie de la géographie. Depuis le début des années 1990, sous l'impulsion d'abord des géographies féministes puis de l'ensemble de la géographie qualitative ${ }^{2}$ anglophone, le corps du/de la chercheur-e est devenu un sujet géographique via la question de la méthode. Dans un double mouvement de dé/re-construction du projet «politique » (i.e. la stratégie identitaire) qui motive l'exercice de la méthode et règle les expériences qui lui sont associées et dont dépend la production des objets scientifiques, les géographies post-modernistes ont renversé le problème du corps. La fin de la fiction positiviste de neutralité esthétique ${ }^{3} \mathrm{du} / \mathrm{de}$ la chercheur-e, soit le principe de la distanciation - l'annulation du corps dans sa matérialité, parce qu'elle est homogène à celle des choses du monde observé, et dans sa subjectivité, parce qu'elle est partielle et irréductible, aux fins de neutralisation des causes de l'altération de la connaissance -, marque l'abandon d'une pensée dualiste de la science qui réclame l'effacement du corps devant le primat de l'esprit pour en faire la condition de production d'une connaissance scientifique vraie. Car le corps n'est pas simplement corps de l'action de la connaissance, mais il est co-impliqué dans l'action de connaissance et cela à deux titres comme corps vivant et comme corps social. Il est à la fois corps cherchant et site d'une subjectivité au travail.

La déconstruction du terrain de la science masculiniste par les géographes féministes anglophones, où le corps est appréhendé sous le prisme de la libido (pulsion de désir) du sujet cherchant et ses modalités expressives comprises comme des formes et des étapes de la relation domination du chercheur sur le contexte de sa recherche, la reconstruction du terrain de la science féministe, où le corps est abordé sous le prisme de l'intersubjectivité et ses modalités expressives traitées comme des formes de la relation de care avec le contexte de la recherche, décrivent toutes deux un corps opérateur de rapports de pouvoir et instrument de consolidation d'identités sociales (genre, sexualité, classe, race, etc.). Cette politisation (politicizing) du terrain fait découler in fine les objets géographiques de processus subjectifsidentitaires et, corrélativement, des formes de l'engagement corporel qui leur est associé. Mais l'approche du corps du/de la chercheur-e avec le point de vue de la positionnalité dans la méthode réflexive - l'objectivation non plus du contenu empirique de la recherche mais de la position (positionality) du sujet-cherchant «situé » au sein de son contexte de recherche pour montrer qu'en tant que corps social il incarne (embodiement) la/les identité/s du/de la chercheur-e et il la/les met en jeu relationnellement (performance) dans le procès de recherche, ne permet pas de dire le tout de la question du corps du/de la chercheur-e au terrain. Elle laisse de côté le corps dans sa réalité vivante et sa participation aux processus de symbolisation des objets du savoir géographique, ainsi que les motifs inconscients qui

\footnotetext{
${ }^{2}$ Je parle ici de géographie qualitative, c'est-à-dire d'une géographie définie par son approche méthodologique, au même titre qu'on a parlé de géographie quantitative. Cette expression témoigne du rôle fondamental qu'a joué la méthode dans le renouveau épistémologique de la géographie féministe, puis post-moderniste en général.

${ }^{3}$ Le terme se réfère ici, et dans l'ensemble du texte, à l'exercice de la faculté d'éprouver, aux expériences qu'elle fonde et à leurs contenus (les éprouvés perceptifs, émotionnels et affectifs d'un corps engagé dans des agirs relationnels), et non pas simplement à l'éprouvé (le jugement de goût) attaché à un type d'expérience en particulier - celle d'un objet d'art - qui constitue historiquement le champ factuel des disciplines de l'art.
} 
installent cette expérience de terrain en situation de transfert et qui en règlent la dynamique de symbolisation.

Si l'idée d'un sujet incarné de la science, dont le corps est siège d'expériences et, via celles-ci, moyen de la construction de l'identité de soi, est devenue un point majeur d'une approche politique de la géographie comme science (politics of doing research), la question afférente de l'esthétique des savoirs géographiques que porte la mise en relation du corps et de la spatialité commence tout juste, quant à elle, à être élaborée. Si la déconstruction du savoir-faire de la science masculiniste a permis de mettre en relation scopicité, comme esthétique de la distanciation, et paysage, comme objet géographique construit dans le regard raisonné, pour mettre au jour un «régime scopique » de connaissance géographique, qu'en est-il du régime esthétique des savoir-faire de terrain contemporains et de leurs objets de savoir géographique ? Le modèle du care qui informe, plus ou moins explicitement, la science qualitative contemporaine contribue-t-elle à substituer une connaissance géographique à fleur de peau à celle d'une science à portée du regard, ou bien la complète-t-elle ?

C'est cette généalogie de la question du corps du/de la chercheur-e au terrain, des positions avancées et de leurs conséquences épistémologiques que je présenterai dans ce texte pour mettre en évidence les apports de la science anglophone contemporaine mais aussi leurs limites conceptuelles et théoriques. Les textes de la géographie française seront ici peu mobilisés, car si le corps, dans le sillage de F. Barthe (2003, 2010), s'installe comme un objet géographique sur le terrain de ses recherches, celui du/de la chercheur-e est encore bien peu interrogé, à quelques exceptions près évoluant entre (r)appels (Pourtier, 1991; Gentelle, 2006), incises (Zeneidi-Henry, 2002) et élaborations (Volvey, 2000, 2004, 2012a, 2012b ; Blidon, 2012).

\section{Le corps du/de la chercheur-e et la politique du terrain dans la géographie anglophone}

Dès le début des années 1990, le problème du terrain surgit dans la géographie anglophone sous la double impulsion du développement d'une épistémologie féministe (Rose, 1993 ; Nast, 1994 ; Duncan, 1996) et des qualitative methodologies (Crang, 2002 ; Limb and Dwyer, 2001; Delyser et al., 2010). Le terrain devient l'objet d'une attention épistémologique. En tant que pratique corporelle à dimension spatiale, il a été travaillé jusqu'à devenir la pierre angulaire de conversions épistémologiques conduites via le déplacement dans le champ scientifique de la problématique des rapports de domination (politics), ainsi que la condition du renouveau « qualitatif» de la science géographique vers son "tournant interprétatif», qui in fine se replie sur le/la chercheur-e dans une démarche réflexive. Il est instauré en pierre de touche d'une définition de la science - sa pratique, ses objets - comme moyen pour conduire une stratégie identitaire (de genre, de sexualité, de classe, de race) au sein de rapports sociaux de domination - c'est l'idée contenue dans l'expression récurrente "politics of the field». Dès le texte fondateur de Rose (1993), le terrain devient, en effet, pour les géographes féministes post-modernistes, la cheville ouvrière de la condamnation de la science dite "masculiniste" et, à partir de Women in the field (Professional Geographer, 1994), il devient l'outil de la refondation d'une science dite « féministe». 


\section{Déconstruire la "performance of power" de la science masculiniste : regard, libido et paysage}

C'est à ce renouveau de la question du terrain que le corps doit d'avoir émergé comme sujet épistémologique. La géographie féministe anglophone a initié une réflexion sur le corps en acte du géographe pour mettre en évidence le fait que la neutralisation du corps dans l'empirisme positiviste en géographie était en fait une ruse d'une science masculiniste visant à cacher les implications identitaires de ses manières de faire avec l'espace et des élaborations théoriques et conceptuelles qui en sont issues. La condamnation de la science classique qualifiée de « masculiniste » (mais bientôt aussi d' « hétéronormative ») s'appuie sur l'examen critique et la mise en rapport de la manière de faire du terrain (le «doing fieldwork»), de la spatialité de celle-ci et de la définition du sujet-cherchant qui la conçoit et s'y engage, pour mettre en relief la ruse («god-trick»-Rose, 1997) objectiviste et universaliste de la géographie positiviste et empiriste. Celle-ci impose une règle de neutralité, de transparence et d'universalité subjective (objectivité), un mouvement de séparation avec l'objet de la recherche (objectivation) réalisé par le truchement de procédures de distanciation, dont les féministes dénoncent le caractère non seulement fictionnel mais stratégique. L'ensemble fonde un régime de connaissance qu'elles dénomment "scopique » (Rose, 1993 ; Nast et Kobayashi, 1996) dont l'objet scientifique, le paysage, est le produit. Elles définissent alors le terrain comme un exercice de domination masculine dont les données sont élaborées en un savoir géographique de type masculiniste. La question du corps est ici au fondement de l'argumentation, puisque l'ordre patriarcal est réputé s'exercer par le biais du corps des hommes et dans le corps des femmes, de même que celle de la sexualité. L'enquête de terrain classique, qui fonde la collecte et la corrélation de données sur l'observation, est ainsi définie par les féministes comme « a performance of power » (Rose, 1996: 58 ; voir Sharp, 2005 - cidessous) où la pratique (work), calquée sur celle de l'exploration, évolue entre possession par l'arpentage, pénétration par le regard et contrôle par le recouvrement exhaustif d'un espace extérieur (field ou land) féminisé (amante/mère), bientôt abandonné aux portes de l'académie sous la double menace des complexes d'Edipe et de castration ${ }^{4}$, pour y être finalement représenté dans l'objet scientifique, le paysage - figure spatiale de la distanciation - (Rose, 1993 et 1996 ; Sparke, 1996; Nast et Kobayashi, 1996). Le paysage est donc, pour les féministes, la traduction en un objet scientifique d'informations empiriques dont les conditions de fabrication et de traitement sont déterminées par un/des motif/s identitaire/s inconscients - un «landscape of power» (Rose, 1993). Rose (1993), mobilisant la critique d'art féministe autour de l'objet paysage et le freudo-lacanisme autour de la pratique de terrain, articule régime scopique et visée identitaire de cette politique du terrain («the masculine active look» / «the feminine position : to be looked at»-ibid.: 102-103) pour en révéler les motifs subjectifs inconscients, soit la stratégie de confirmation ou consolidation de l'identité sociale sexuelle - masculine et hétérosexuelle - du chercheur (voir aussi Sparke, 1996).

«Some cultural geographers suggest that the discipline's visuality is not simple observation but, rather, is a sophisticated ideological device that enacts systematic erasures. They have begun to problematize the term 'landscape' (...), and they have argued that it refers

\footnotetext{
${ }^{4}$ Les complexes permettent de construire le regard du chercheur (homme, blanc, hétérosexuel) comme «a gaze torn between pleasure and its repression » (Rose, 1993: 103) et la pratique d'écriture comme de la sublimation : «Domination of the land began to be seen as both incest and rape, and the horror of this necessitated a psychological and emotional separation from the land and from woman. » (ibid.: 115).
} 
not only to the relationships between different objects caught in the fieldworker's gaze, but it also implies a specific way of looking. (...) [A]s a consequence, they argue that the gaze of the fieldworker is part of the problematic, not a tool of analysis. Indeed, they name this gaze at landscape a 'visual ideology', because it uncritically shows only the relationship of the powerful to their environment. This is an important critique of the unequal social relationship implicit in one element of geographical epistemology.

Questions of gender and sexuality have not been raised by this newer work, however. (...) The feminization of what is looked at does matter, because it is one half of what Berger characterizes as the dominant visual regime of white heterosexual masculinism: 'women appear', he says, but 'men act'. This particular masculine position is to look actively, possessively, sexually and pleasurably, at women as objects. (...)

The recurring but uneasy pleasure that geography finds in landscape, acknowledged but never addressed, is a version of the discipline's aesthetic masculinity. (...) I will draw on the arguments of feminists working with Freud and with Lacan's re-reading of Freud, because several have focused on the contradictoriness of 'sexuality in the field of vision' through these forms of psychoanalysis. (...) This section argues that these feminist psychoanalytic commentaries offer eloquent critique of geography's white, heterosexual, masculine gaze, a gaze torn between pleasure and its repression. » (Rose, 1993: 87-88 et 99-103).

La géographie féministe déconstruit à travers l'analyse des différents éléments inconscients - fantasmatiques et agis -, du rapport entre corps et spatialité, la masculinité et l'hétéronormativité d'une science: elle fait de la géographie, dans sa fabrique même, une discipline de la reproduction sexuée et sexuelle des rapports de domination entre genres, repérable aussi bien dans son engagement que dans les modalités d'organisation duale de la spatialité de ses tâches (entre terrain et académie/bureau, entre procédures arpentantes et pénétrantes de terrain et procédures distanciantes d'écriture), que dans ses objets scientifiques.

\section{L'embodied knowledge de la science féministe : le travail de l'intersubjectivité}

« Fieldwork has often been critiqued by feminist geographers as a masculinist tradition (e.g., Rose, 1993). However, the field is an ambivalent place for feminist work: holding the potential for misrepresentation and the inappropriate performance of colonizing power relations, while at the same time presenting the possibility of meeting and opening a true dialogue with those so often marginalized and silenced by dominant discourses and representations (...). (...) One obvious way of developing a critical feminist politics within a range of methods is collaborative research, from the conception through the outcomes of research. » (Sharp, 2005: 306).

A contrario, l'enquête de terrain féministe (feminist methodology) a été placée au fondement d'un "feminist political project within the discipline » (Sharp, 2005: 304), jusqu'à participer parfois d'une stratégie d'activisme politique (Kobayashi, 1994 - voir l'ensemble du numéro thématique The Professional geographer, 1994). Le motif cognitif se trouve assujetti ou couplé au motif politique (" the concept of fieldwork as resistance », Nast, 1994: 60) : la consolidation ou la construction identitaire individuelle et/ou collective des minorités opprimées (selon les catégories de genre, race, classe, etc.), le renversement de l'ordre patriarcal et l'avènement de la fin de l'hétéronormativité en géographie.

«Over the past decade, (...) I have engaged in political efforts to align my academic objectives with community activism, in fact, merging field research and activism to the point 
where I could not distinguish the two. (...) If one's objective is to combine the political and the academic, such committed fieldwork is very effective. » (Kobayashi, 1994: 73-74).

Un « to do gender » dont les féministes ont discuté depuis la réalité et la consistance épistémologique (Sharp, 2005). Ainsi, même si les auteur-e-s féministes ne sont pas les seule-s à avoir participé à la refondation épistémologique du terrain qualitatif, leurs élaborations l'ont décisivement informée (Sparke, 1996 ; voir aussi Limb et Dwyer, 2001) en enracinant les méthodes de terrain qualitatives contemporaines dans des expériences et des pratiques qu'elles/ils rapportent aux relations des femmes, et en particulier des mères, à leur environnement de vie. H. Nast (1994) dans son introduction à Women in the field énonce l'enjeu politique de la méthode pour les féministes, avant d'en donner les principes :

« The papers indicate that feminist scholars engage in fieldwork as a means of resisting patriarchy and other forms of domination in ways that are congruent with women's experiences. » (Nast, 1994: 61).

Ces principes précisés ailleurs dans son texte dessinent les contours d'un modèle féministe de terrain fondé sur le care :

"The social connectedness of women to others carried in everyday practices has fostered ways of knowing or epistemologies that are different from those of the men. Women have typically been nurturers and caregivers trained in the art of listening and otherempowerment. » (ibid.: 55).

En faisant le portrait du/de la géographe de terrain en caregiver, les auteur-e-s féministes insistent sur les manières de faire avec attachées à ce modèle de terrain substitutif, manières relationnelles conçues pour installer la «supportive atmosphere » (Duncan, 1996 ; Rose, 1996; Kneale, 2001) ou le «facilitating environment» (Bingley, 2003) réputés caractériser la méthode intersubjective qu'elles préconisent.

« The politics of involvement, for feminist researchers, require research methods that recognize the relationship with others as one of (ideally) mutual concern and trust. (...) Feminist methods that stress mutual respect and involvement, shared responsability, valuing difference, and nonhierarchical ways of achieving ends are not simple or shallow gestures accommodation, nor are they just an alternative methodology. Such methods define an approach to political change. » (Kobayashi, 1994: 76).

Elles/ils tendent à distinguer les spatialités des faire masculiniste et féministe en opposant la distance (distanciation) et la proximité (co-implication et compréhension dans l'intersubjectivité) (Sharp, 2005). Elles/ils définissent alors le faire de terrain comme « betweeness » ou « withness » (Nast 1994 ; Rose 1996 ; Bondi 2003 ; Bingley 2003), un faire avec par opposition au faire (de)dans/dehors de la géographie masculiniste et décrivent le terrain en termes de «space of betweenness » ou "place in-between » (Katz, 1994 ; Duncan, 1996; Cupples, 2002; Avis, 2002), le dotant d'une qualité d'intermédiarité (espace (de 1')entre-deux - «the in-between quality of [that] space » - Avis, 2002: 206).

«Gillian Rose (...) discusses the creation of new forms of space based on 'all envelopping' notions of 'between' and 'around' - spaces that are supportive and enabling in contrast with notions of 'distance and separation' that affirm an individualistic 'master identity’. » (Duncan, 1996: 6).

Ces manières de faire féministes et leur spatialité s'articulent à une autre corporalité de terrain. Le corps agissant, dans ses multiples dimensions, est mobilisé comme opérateur de l'intersubjectivité aux fins de production de données de terrain. Ainsi, certain-e-s chercheur-e- 
$\mathrm{s}$ appuient leur effort théorico-méthodologique sur la psychothérapie humaniste ${ }^{5}$ pour faire de l'empathie et de l'identification un outil technique de l'entretien qualitatif supposé réduire la distance qui sépare le sujet-cherchant et le sujet-enquêté (Bondi, 1999, 2003 ; Aitken et Herman, 1997 ; Kneale, 2001 ; Bingley, 2003).

« This paper introduces psychoanalytic conceptualisations of identification and empathy as ways of thinking about fieldwork interactions. I argue that these ideas have considerable relevance for feminist geography as resources for reflecting on relationships between researchers and those they research, especially in relation to debates about power and positionality in qualitative fieldwork. (...) I argue that empathy can be thought of as entailing an oscillation between observation and participation which creates psychic space or room to manoeuvre, and that it provides a way of understanding other people's experiences in the context of both similarities and differences between researchers and research subjects. » (Bondi, 2003: 64).

D'autres, redécouvrant la problématique du jeu (playing), instaurent le partage de situations de vie (Aitken, 2001) ou le dispositif artistique en cadres de la relation de recherche. Un développement présenté par Lloyd et al. (2012) en ces termes : "We discuss how the process of sharing knowledges is based on special and intimate relationships and connections, learnings and transformations.» Punch (2001), par exemple, traite de la préparation commune des repas, du fait de dormir dans la même chambre que les familles qu'elle enquête et de jouer avec les enfants de ces familles comme des procédures pourvoyeuses de données scientifiques en Bolivie du sud où elle conduit ses recherches de terrain. Cupples (2002) prend en charge la question des rapports de pouvoir liés à la sexualité du/de la chercheur-e et renverse le tabou de l'activité sexuelle sur le terrain pour arguer de son potentiel relationnel. Tandis que Longhurst et al. (2008) transforment la consommation d'un repas en commun en méthode d'enquête sur les migrants en Nouvelle Zélande. Enfin, au croisement de ces nouvelles procédures, Bingley (2003) applique la technique winnicottienne du holding dans son protocole méthodologique inspiré de l'art therapy pour supporter un travail de groupe sur la dimension genrée du rapport au paysage. Toutes ces techniques du corps placées au principe de l'intersubjectivité dont procèdent les données de recherche appellent donc une esthétique contraire à celle associée au terrain masculiniste - une esthétique du contact.

«The examples I use above about knowledge building through embodiement belie a certain kind of researching body (...). » (Parr, 2001: 162)

Dans un premier temps, les auteur-e-s féministes participent ainsi et de manière paradoxale à la fondation méthodologique du «linguistic turn», un des moyens de l'«interpretive turn ». Car au croisement des pratiques d'ethno-méthodologie et des pratiques d'enquêtes de groupe (les in-depth et les focus groups), cette nouvelle corporalité de terrain sert surtout à faire procéder la parole.

«Part and parcel of ethnographic research is participating in social situations. In the encounters excerpted from my research diary, I was an embodied observer, not a detached one.

\footnotetext{
${ }^{5}$ Notamment, C. Rogers (1902-1987), psychologue états-unien, met au fondement de la clinique la relation entre le patient et le thérapeute, notamment la forme d'attention (écoute empathique, authenticité et non jugement) du thérapeute au patient. Et D. W. Winnicott (1896-1971), psychanalyste anglais, est un des théoriciens de la psychanalyse de la relation d'objet et le fondateur d'une théorie relationnelle de la psychogénèse et de la clinique analytique, organisée sur le principe du care.
} 
(...) During covert ethnographic research in parks and streets around the city of Nottingham, my strategies of access involved different kinds of 'making' of my body. (...) My intention was not to 'pose' as someone with mental-health problems but to find ways to make some kind of subtle 'body talk' with those users of public space who did face such challenges, hoping that this would lead to more verbal relationships. » (Parr, 2001: 161)

Quels que soient la méthodologie privilégiée, le type d'approche et de dimension corporelles mobilisé, la facilitation par le corps de la relation de recherche permet, selon les féministes et les qualitativistes en général, de «listening to, giving voice to and representing the silenced $\gg$ (Crang, 2002: 648) pour donner lieu à un matériau de type discursif, représenté dans les textes scientifiques et assurant l'empowerment reciproque du/de la chercheur-e et du sujet-enquêté. Ces productions académiques mêmes peuvent être le fruit d'un travail collaboratif qui pousse la logique du faire avec jusqu'à la représentation de toutes les voix dans un discours académique et prennent des formes variées aussi bien textuelles que cartographiques (Maulion, 2008) ou graphiques (Kneale, 2001). C'est la métaphore du panier tressé que Lloyd et al. (2012: 514) choisissent de mettre en entame de leur texte qui rend compte du processus collaboratif de «co-authorship » en situation de vie (entre chercheur-e-s non-indigènes et un groupe de femme indigènes en Australie), pour dire la collaboration et les processus de transformation de soi et de l'autre, de soi avec l'autre, en celle-ci.

En effet, la référence freudo-lacanienne que ces auteur-e-s mobilisent (cf. Rose, 1993 - ci-dessus ; Bondi 1999 - ci-dessous) vient appuyer le rapport établi entre langage et identité sociale $^{6}$, et contribue à évacuer la part pré-verbale de la donnée de terrain acquise en ces méthodes intersubjectives à fondement corporel et empêche, au-delà, que soit pensée la part corporelle de cette donnée élaborée dans le savoir géographique. Faciliter l'expression verbale de ceux qui n'ont pas la parole, représenter leur discours dans un texte sont, pour eux/elles, les outils méthodologiques de la résistance politique à et du renversement de la patriarchie. Au-delà, la problématique de la construction de l'identité sociale scientifique devient une question de représentation de soi dans/par le discours (entretiens de terrain, conférences ou textes) (Bondi et al., 2002) et celle de l'autorité scientifique devient alors une question d'autorat - soit une double «politics of representation » (Nast, 1994). Les féministes (Avis, 2002) discutent ainsi de «the gendered nature of voicing the self» pour associer, du côté du paradigme masculiniste de la géographie classique, neutralité-universalité du sujet cherchant et choix de celui-ci de taire («left invoiced») son identité, et du côté du paradigme féministe, travail de positionnalité et choix de dire («spoken ») son identité.

Cette question de la représentation de soi/de l'autre dans le discours scientifique fonde la méthode réflexive (Rose, 1997), jusqu'à en faire la condition même de la validité d'un régime de connaissance féministe (et post-moderniste en général). Si neutralité et distanciation ne sont plus placées au fondement d'un savoir positif, si le terrain est replié sur des stratégies de consolidation identitaire au sein de rapports sociaux de domination, si le

\footnotetext{
${ }^{6}$ La conception lacanienne de l'identité subjective comme assujettissement à un ordre symbolique par le truchement du langage et via des identifications, a informé le féminisme anglophone et l'approche de la question de l'identité sexuelle qu'il a élaborée dans la problématique de la positionnalité. Non seulement le sentiment d'unité de soi est une illusion, mais le sujet se trouve actuellement divisé entre la multiplicité de ses identifications aux systèmes symboliques qui le déterminent (en le nommant), l'aliènent et l'identifient d'être tel ou tel. L'idée que «le langage est cause du sujet» a entraîné l'assimilation de l'appareil psychique et de l'appareil du langage. Le rapport établi entre langage et identité sociale peut ainsi expliquer la profondeur de l'articulation entre empowerment et linguistic turn chez les féministes, dont l'enjeu concerne autant le sujetenquêté que le sujet-cherchant.
} 
savoir qui en élabore et formalise les données est défini comme «situé » («situated knowledge ») - c'est-à-dire non seulement non universel mais lié à un point de vue identitaire au sein d'un rapport de pouvoir-, alors seule l'objectivation de cette «position» (positionnality) et du contexte d'interactions où elle joue devient garante de la validité du savoir construit. Tandis que, la conception du terrain comme "field-as-betweeness » conduit à une compréhension relationnelle et processuelle du terrain (l'idée de negociation), dans laquelle la situation de recherche est condition de co-émergence et de co-construction des identités (sujet-cherchant/sujet-enquêté). L'intersubjectivité impliquée par la situation de terrain a des effets identitaires pour/sur les acteurs qui s'y engagent. Entre introspection et analyse de la relation aux sujets-enquêtés, toutes deux construites dans le texte (article, ouvrage, communication), la méthode réflexive permet d'atteindre la «position» du chercheur dans la situation de rapport de domination qu'est le terrain et elle permet de rendre compte de la manière dont cette «position» informe le savoir «partiel» qu'il produit (McDowell, 1992 ; Moss, 1995).

Cependant, si la relationnalité, abordée dans un premier temps dans sa dimension interdiscursive, est productrice d'un matériau linguistique représentable dans un texte scientifique, le modèle du care, choisi pour ces pratiques et le type d'engagement qui lui est lié, est bel et bien responsable du surgissement de la problématique du corps dans l'épistémologie féministe, à la fin des années 1990. Les féministes interrogent la place du corps dans la politique de la recherche et tendent à définir le terrain comme une situation où des corps co-présents interagissent et, ce faisant, (re)produisent de l'identité. Le corps socialisé, engagé dans la relation créée par la méthode, est considéré à la fois comme le site, le marqueur et l'opérateur de l'identité sociale ou des identités sociales, au sein de la situation de recherche (Nast, 1998 ; Parr, 2001 ; Sharp, 2005 ; Longhurst et al., 2008). Le corps social situe l'identité et devient le lieu de l'interaction de recherche. Comme l'écrit Rose (1997: 315) : "researching gender is 'doing gender'. (...) Our identities do not pre-exist our performances of them. " Les manières dont les identités sociales sont corporellement engrammées (embodied), représentées (mapped) dans les corps, mises en jeu et reconstruites (performance of identities) dans la pratique relationnelle de terrain deviennent un champ d'application de la méthode réflexive. Celle-ci gagne et occupe alors le terrain du corps (Rose, 1996, 1997 ; Nast, 1998 ; Parr, 2001), qui, dans sa dimension symbolique, devient objet de la méthode visant l'objectivation d'une connaissance située (la «situated knowledge » est d'abord une « embodied knowledge »).

«It made me rethink fieldwork and how our bodies might be considered places where we field or draw in difference, or as sites where difference is placed. In effect, bodies are physical sites upon which the world inscribes itself - places to which others come and mark their difference, places like any other place - localized and with continuously negociated boundaries and subregions. » (Nast, 1998: 95)

Par ailleurs, au milieu des années 2000, cette considération pour les corps interagissant dans le procès d'une recherche aux méthodes relationnelles a conduit les féministes, et audelà, l'ensemble des géographes qualitatifs, à la reconnaissance d'un matériau de terrain préverbal (ou pré-linguistique) et à interroger, en conséquence, sa représentation scientifique (processus, modalités et formes). C'est ce dont se saisit alors le courant de l'emotional geography (Davidson et al., 2007) - dans lequel l'on retrouve notamment L. Bondi (2005). C'est aussi ce que Crang (2003), privilégiant une modalité particulière de la corporalité 
impliquée par les méthodologies qualitatives - le toucher -, interroge sous le terme générique d' " haptic knowledge », lançant dans la géographie de langue anglaise l'idée d'hapticité développée depuis par Paterson (2009 ; Paterson et Dodge, 2012) mais dans une perspective moins épistémologique que cognitive - haptic knowledge renvoyant aux savoirs spatiaux de catégories de populations souffrantes ou handicapées.

« However, there is rather less on the actual processes of learning through our bodies' responses and situations - that is, haptic knowledges » (Crang, 2003: 499).

Ce texte de Crang (2003) est le deuxième d'une série de trois textes publiés dans Progress in human Geography, dans lesquels il propose une synthèse des méthodes et questionnements de la «new orthodoxy » qualitative, toutes orientations confondues. Il y met en scène le passage de méthodes («verbal approaches/methodologies») fondées sur l'interdiscursivité ( oral methods ») et l'analyse de discours - la nouvelle orthodoxie du linguistic turn donc-, à des méthodes fondées sur la présence physico-émotionnelle, l'engagement du corps («corporeal performances ») et les interactions à travers le corps (« embodied interactions »), posant l'idée d'une « geography as embodied work».

Il est alors possible de retracer la trajectoire épistémologique de la géographie postmoderniste sur la question du corps : d'une part, d'un corps outil de l'activité de recherche (researching body) utilisé pour réduire la distance et l'autorité, à un corps incarnant l'identité subjective au travail, opérateur et processeur de données identitaires construites dans le savoir géographique (embodied knowledge/geography); d'autre part, du régime scopique de connaissance que la géographie féministe a déconstruit au début des années 1990, à un régime discursif («discursive regime »-Crang, 2003: 499) qu'elle a grandement contribué à mettre en place à la même époque, aux interrogations contemporaines de la géographie qualitative sur un possible régime haptique ${ }^{7}$. La question des régimes esthétiques de connaissance en géographie est désormais ouverte, interrogeant tant la représentation de données pré-verbales et non scopiques dans le savoir géographique, les objets associés à chacun des trois régimes de connaissance géographique, que l'articulation entre ces derniers, et posant le problème du cadre théorique à l'intérieur duquel penser le régime haptique et son articulation aux autres régimes de connaissance.

\section{Corps, spatialité, identité : quelle esthétique des savoirs géographiques ?}

\section{Identité sociale et modèle relationnel de terrain: désaccords métathéoriques}

La définition relationnelle du terrain renvoie au modèle du care, bien repérable dans les citations présentées plus haut dans le texte, la théorie féministe du terrain mobilisant tant son vocabulaire (caregiver, playing, etc.), que son sens des gérondifs (The Professional geographer, 1994) et ses idées (le terrain comme betweeness et son rapport avec la performance). Cependant, sauf chez Bondi (1999, 2003), Bingley (2003), Aitken et Herman (1997), Aitken (2001), Kneale (2001), les références sous-jacentes à ce modèle et à son usage sont rarement objectivées et réfléchies. A l'exception du texte de Vivat (2002), l'horizon référentiel de cette théorie féministe ne renvoie pas, à ma connaissance, aux théories du care de la philosophie du droit de Carol Gillian ou de Joan Tronto des années 1980, mais plutôt

\footnotetext{
${ }^{7}$ C'est-à-dire lié au toucher dans ses dimensions motrice, sensorielle et émotionnelle (tactilité et empathie).
} 
aux psychothérapies humanistes des années $1960-70^{8}$ (voir Social and Cultural Geography, 2003). Comme pour l'éthique du care, il s'agit d'une théorie des relations intersubjectives entre deux pôles : l'autre, considéré dans sa vulnérabilité (voire sa dépendance) - ici les minorités sociales -, et le caregiver, reconnu dans ses dispositions (attention à l'autre et réceptivité, responsabilité à son égard et souci de sa situation, non jugement) et sa capacité pratique (les tâches accordées avec ses dispositions et ajustées aux besoins de l'autre) - ici le/la chercheur-e -, qui trouve son modèle dans la relation entre l'environnement maternant et le nourrisson. Cette refonte théorique du terrain sur le modèle du care pose plusieurs questions: le risque du maternalisme qui limiterait l'étendue de la refondation épistémologique; la difficulté qu'il y a à accorder les définitions de l'identité subjective portées par le féminisme (identité sociale) et par les pensées humanistes (identité narcissique); l'évacuation du spatial au profit du social dans une théorie positionnelle; le défaut d'analyse symétrique et critique de cette refondation épistémologique à partir d'un tel modèle de terrain. Je reviendrai ici sur les trois derniers points (pour plus de détails voir Volvey, 2012b), pour montrer combien c'est le manque d'investissement pour raisons politiques, du corps comme donnée vitale de la réalité humaine et de la théorie transitionnelle en psychanalyse ${ }^{9}$ - qui pourtant fonde la méthode -, qui est responsable in fine du ratage de l'esthétique haptique des savoirs géographiques et au-delà de la question esthétique en épistémologie de la géographie.

Le champ référentiel de la géographie féministe en matière de terrain est ce que Bondi appelle la "humanistic psychotherapy » et à laquelle je me réfère pour ma part en tant que psychanalyse transitionnelle (Volvey, 2004, 2012b, 2013). Or cette dernière promeut une définition narcissique de l'identité qui est difficilement accordable avec la visée politique de cette refondation méthodologique qui vise la consolidation ou la construction de l'identité sociale du sujet-cherchant et du sujet-enquêté. L'irréductibilité de ces différentes acceptions philosophiques de l'identité subjective ${ }^{10}$ est pourtant au cœur d'un essai biographique de Bondi qui raconte combien, dans les années 1990, son engagement scientifique dans une géographie féministe d'inspiration lacanienne, centrée théoriquement sur les questions des identités sociales fragmentées, s'inscrivait en décalage d'une expérience personnelle de cure psychanalytique, d'inspiration humaniste (cf. note 5), centrée sur la question du «sense of self $\gg$ (l'identité narcissique) :

\footnotetext{
${ }^{8}$ Bondi (2003) fait directement référence aux pratiques thérapeutiques inspirées de D. W. Winnicott (2005 [1971]), mais aussi de Carl Rogers (On becoming a Person: A Therapist's View on Psychotherapy, 1961) et de Kenneth Wright (Vision and Separation: Between Mother and Baby, 1991). Bingley (2003), Aitken et Herman (1997), et Aitken (2001) font, pour leur part, directement référence à Winnicott.

${ }^{9}$ L'expression de psychanalyse transitionnelle que j'emploie est une extrapolation à l'ensemble du domaine théorique de la psychanalyse des personnalités dites narcissiques-identitaires et des états-limites, que je fais, des élaborations métapsychologiques de Winnicott (2005 [1971]), dont le concept clé est "phénomène transitionnel », et des propositions cliniques de R. Kaës et al. (1997).

${ }^{10}$ La définition narcissique de l'identité met en équivalence la subjectivité et l'égoïté : le sujet est une entité indépendante et constante, donnée dans l'expérience, connue de manière réflexive et discursive - processus dont découlent l'amour ou l'estime de soi (le narcissisme) - et placée au fondement de la conscience et de la revendication de soi (l'identité). Tandis que la définition de l'identité subjective comme sociale (cf. note 6) est liée à une compréhension de la subjectivité comme sujétion, pour en faire le produit des aliénations successives (des identifications) de l'individu à des systèmes symboliques variés portés par des institutions - au nombre desquelles la famille, la différence de sexes, etc. Sur les différentes définitions de l'identité voir, par exemple, Balibar, Cassin et Libera (2004: 1250).
} 
« Over a period of several years I saw a therapist trained within a broadly humanistic therapeutic orientation. At much the same time I was engaging academically with feminist poststructuralist work and with feminist debates about psychoanalysis. To put it crudely, by day I read, talked, and wrote about decentred selves, fragmented identities, and so on, and then out of work-time in a quite separate space I explored my sense of self within a therapeutic frame in which notions of authenticity, genuineness, centredness, and so on came into play. (...) So, while I read about psychoanalysis and especially about Lacan's anti-humanistic return to Freud, I rather avoided reading about humanistic therapies or about the influence of humanistic philosophies on psychoanalytic theory and practice. The separation was therefore a double one, both between theory and practice, and between anti-humanism and humanism. (...) the boundary I maintained between work and therapy was also one between humanism and antihumanism. » (Bondi, 1999: 15).

En conséquences, elle y appelle de ses vœux l'installation du courant humaniste dans la géographie, pour soutenir notamment sa conversion qualitative :

«Like proponents of humanistic perspectives and qualitative methods in geography, those writing within this psychotherapeutic tradition drew inspiration from a range of humanistic philosophies including phenomenology and existentialism. In the context of these common influences, the absence of reference to the humanistic psychotherapy movement in geographical discussions - especially in texts concerned with methodological practice rather than epistemological underpinnings - is, I think, notable. (...) Further, this silence has become more striking as the discipline has engaged with psychoanalytic theory in a manner at some considerable remove from contemporary psychotherapeutic practice. » (ibid.: 17).

En revanche, elle n'interroge pas les problèmes philosophiques posés par cette installation qu'elle réalisera pour sa part, on l'a vu, en complément technique des méthodologies féministes qualitatives d'entretien - «as resource[s] for reflecting on fieldwork relationships negotiated in the course of feminist geography » (Bondi, 2003: 64). Ainsi, elle mobilise une technique thérapeutique qui a à voir avec le problème de l'identité narcissique pour participer, dans le champ de la géographie féministe militante, à la construction scientifique de l'identité sociale de genre. Cette difficulté métathéorique étant un impensé d'origine politique, Bondi n'envisage pas les moyens pour la réduire. On retrouve le même problème chez Bingley (2003) et chez Avis (2002).

De fait, le problème soulevé par Bondi (1999) ne se résout pas dans un simple départage entre lacanisme et winnicottisme (ou théorie transitionnelle en général), départage par lequel le lacanisme contiendrait la théorie (ou une partie de) de l'identité subjective et le winnicottisme les moyens cliniques d'y accéder. On ne peut pas, à mon sens, mobiliser la pratique clinique transitionnelle pour travailler sur les dimensions de l'identité subjective définies à partir de Lacan, sauf à considérer que la transitionnalité n'est pas une matrice psychanalytique mais un ensemble d'ajouts de concepts à un corpus théorique existant (freudo-lacanien) et surtout de techniques. «L'apport de Winnicott va au-delà d'un simple raffinement de notre compréhension du psychisme, il révolutionne celle-ci » (Roussillon, 1999:10) : la différence entre lacanisme et transitionnalité joue en profondeur autour de la définition de la question de l'identité subjective, et partant de la souffrance psychique ${ }^{11}$, de

\footnotetext{
${ }^{11}$ D'un point de vue étiologique, la souffrance narcissique-identitaire renvoie à des dysfonctionnements précoces de la relation environnement et nourrisson (du care et du playing ) qui ont fait entrave au développement normal de la créativité (primo-symbolisation). Elle se manifeste par l'inscription ou le rejeu dans l'actuel des traces des ratés de la primo-symbolisation - ratés portant précisément sur le problème de l'unification de la multiplicité et de la diversité des états subjectifs (je) du nourrisson sous une construction symbolique identitaire suffisamment cohérente, consistante et continue (je-moi), placée à la base de la différenciation, de l'expression et de la revendication de soi.
} 
l'inconscient $^{12}$, de la question du transfert et de ses enjeux subjectifs ${ }^{13}$, ensemble de définitions dessinant une autre anthropologie psychanalytique, dont l'aménagement de la pratique clinique est une conséquence - même si elle en a été le déclencheur (pour plus de détail voir Volvey, 2012b). Ainsi, quel est le «sense of self» qui émerge des dimensions relationnelles et performatives du terrain, quel est ce «care for the self» qui s'étend dorénavant au géographe de terrain reconnu-e dans sa vulnérabilité et sa souffrance psychique (Myers, 2001 ; Bingley, 2002 ; Sharp, 2005), mais aussi quelle est cette « search for the self » (Myers, 2001 ; Avis, 2002), dont parlent les géographes féministes et au-delà l'ensemble des géographes qualitativistes dans leurs textes méthodologiques, autant que dans leurs compte rendus biographiques de terrain (Myers, 2001) ? De quel problème identitaire s'agit-il, mais aussi de quelle fonction inconsciente du terrain comme pratique de créativité scientifique sur le fondement d'une expérience relationnelle à dimension corporelle ?

La question de la spatialité de la pratique de terrain fondée sur le modèle du care, dont on a vu qu'elle était un argument des féministes contre le masculinisme (proximité contre distance), qu'elle était très présente dans le discours descriptif de la méthode féministe, est finalement peu investie par les féministes et plus généralement les qualitativistes. La tendance est à une conception métaphorique de l'espace (comme espace dialogique dans le régime linguistique, comme espace intrapsychique dans le régime haptique), et à une évacuation de la dimension spatiale de la pratique et de l'expérience qui lui est associée. C'est une conséquence lourde des théories de l'identité en termes de positionnality, qui replient la question spatiale sur le social et traitent l'espace métaphoriquement - et non pas comme une dimension du social. La pratique de terrain devient exclusivement un engagement du/de la chercheur-e avec les sujets-enquêtés dont i1/elle est séparé-e, au sens où il/elle est différent-e en termes d'identité sociale - la pratique est «socially engaged». L'espace n'a finalement pas d'autre actualité qu'en tant que lieu (field) où se passe la relation (work) entre des sujets qui sont les « sites » de la signification (identitaire), il n'est pas ce avec quoi le/la chercheur-e de terrain fait via un engagement dont la spatialité, puisqu'elle est agie, participe à la construction de l'identité sociale qui à son tour se manifeste spatialement.

«I draw on the object relations tradition of psychoanalysis, within which psychic interiors are understood as richly peopled - filled with representations of selves and others and parts thereof. (...) With the two interconnected concepts of identification and empathy (...) I offer a perspective on subjective experiences within fieldwork relationships (...). I argue that empathy provides space for difference, while also enabling the researcher to communicate respect and recognition. First, (...) identifying with another presupposes the separateness of self and other, but also suggests that the boundary between the psyches of self and other is permeable rather than impermeable (...). Identification is therefore a concept that represents the spatiality of self and other in terms of flexible and open boundaries together with movement

\footnotetext{
${ }^{12}$ L'inconscient n'a pas la définition d'un système constitué de contenus psychiques : représentants de la pulsion qui échappent aux deux autres instances de la topique freudienne ou signifiants du « discours de l'autre » de la position lacanienne. Eprouvés non symbolisés formant les traces d'une incomplétude de la symbolisation primaire (soit, les non-advenus de la créativité) chez Roussillon (1999), ou bien symbolisations primaires défaillantes (soit, les défauts de la créativité) chez Anzieu (1995).

${ }^{13}$ Le transfert devient transfert d'une situation originaire historique sur la situation relationnelle actuelle trouvée/créée avec le cadre. Roussillon parle de «transfert de situation », expression par laquelle se trouve poser le principe d'une fonction transitionnelle du cadre et de l'analyste, qui met l'environnement-objet et le corps au centre du processus clinique, pour faire advenir créativement le non advenu de la symbolisation/subjectivation ou pour réparer les formes primaires défaillantes de l'identité narcissique.
} 
across boundaries. (...) processes of identification help to produce the spatiality of psychic interiors » (Bondi, 2005: 68-69).

Malgré l'inflation des notions spatiales, la dimension spatiale des phénomènes transitionnels demeure non élaborée, parce qu'impensée tant chez les psychanalystes que chez les géographes. Le fait qu'il n'y ait pas d'attention portée aux faire avec l'espace de soin et aux agir relationnels qui constituent ce faire dans les textes anglophones qui mobilisent cette théorie psychanalytique pour penser les conditions du terrain, fait contresens en psychanalyse transitionnelle - la spatialité relationnelle ne renvoie pas qu'à des échanges intra- et interpsychiques de contenus symboliques en situation de care ("playing is doing » écrit Winnicott - 2005 [1971]: 55 - contre M. Klein) - et problème en géographie - comme science de la dimension spatiale du social. La différenciation subjective passe par le corps pour autant que celui-ci est noué à l'espace par des agir relationnels, pour autant qu'il est opérateur de spatialité.

La géographie féministe qualitative n'a pas fait, à ma connaissance, le travail critique qui consiste à retourner sur ses propres propositions théoriques les outils d'analyse qu'elle emploie sur ses sujets d'enquête. Elle n'a pas appliqué le principe de symétrie qui lui permettrait d'envisager vraiment les éléments d'un régime de savoir haptique au cœur de la pensée géographique - contrairement à ce qu'elle a fait autour de la géographie masculiniste. On peut d'ailleurs faire l'hypothèse qu'elle enferme la transitionnalité dans la méthodologie pour ne pas travailler la question des motifs inconscients qui accompagnent la recherche qualitative aujourd'hui et informent la créativité scientifique contemporaine, ce qui aurait alors deux implications fortes, d'une part remettre l'inconscient de terrain géographique sur le métier, d'autre part, questionner les fondements catégoriels identitaires de leur constructivisme. Ainsi, Rose (1997), après avoir fait référence à l'inconscient qui œuvre au cœur de la relation intersubjective et qui joue un rôle dans la co-construction identitaire, invoque la nécessité de mettre des limites à la méthode réflexive pour ne pas tomber dans la « goddess-trick» du désir de mise en transparence des sujets et de leur champ d'interaction, et tient curieusement l'inconscient du/de la chercheur-e hors du champ de la méthode réflexive.

«In this argument, we depend for our sense of self precisely on an otherness we can never fully know. Moreover, as Madge (...) suggests, some understandings of that relationality emphasize the role that fantasies about the researched as 'other' may play in the research process. Madge draws on the work of a number of postcolonial theorists to suggest that the relation between researchers working in the third world and their research subjects may be structured by (among other things) boundaries constructed by psychic processes such as fetishism and paranoia; as Pile (...) notes, such processes, dependent as they are on the unconscious, are not possible to access fully. » (ibid.: 314 ).

Cette absence de symétrie autour d'un inconscient de terrain qui met en jeu relationnellement autant le sujet-cherchant que le sujet de la recherche, est incarnée de manière exemplaire dans ce texte de Bondi (2003: 67) :

«Conversely, research relationships may have effects that research respondents experience as beneficial and therapeutic. (...) Put another way, researchers would surely be in breach of (implicit or explicit) codes of ethical conduct if they suggest that participation in research relationships would yield (or be likely to yield) therapeutic benefits, just as psychotherapists would breach the ethical frameworks guiding their work if they displaced their clients' capacity to make meaning with their own (...). ». 
Elle se joue en effet à deux niveaux, selon deux déplacements. Si Bondi compare la situation de terrain et la situation clinique compte tenu de ce qui s'y joue dans la relation autour de la dimension inconsciente de la personne et des techniques mises en œuvre, c'est pour mieux les séparer. Elle réaffirme d'une part, la primauté du projet cognitif du chercheur sur un éventuel objectif thérapeutique (et inversement pour le thérapeute), elle n'envisage, d'autre part, l'effet secondaire (« side effects ») thérapeutique du terrain scientifique que pour le sujet-enquêté. Ce faisant, elle met de côté le fait que la situation de recherche puisse être une situation de transfert non pas (seulement) pour le sujet-enquêté qui y participe, mais (surtout?) pour le sujet-cherchant qui la trouve/crée, et dans laquelle œuvrent des motifs inconscients propres au chercheur. Chez Bondi, comme chez Bingley (2003), le géographe de terrain est habillé des qualités du praticien transitionnel qui met en place un dispositif transitionnel pour faire des enquêtes, tandis que ses motifs non cognitifs ne sont pas interrogés (pas même, sous la forme du contre-transfert). Si avec la critique d'inspiration lacanienne du terrain masculiniste, l'identité subjective n'était plus placée dans la perspective du et encodée par l'impératif cognitif, et si sa part inconsciente avait été révélée comme œuvrant au cœur de la recherche, motivant son projet et informant le savoir, on voit que les géographes féministes ont quelques difficultés à considérer ce qui motive et informe inconsciemment leur recherche, qui peut s'analyser dans leurs pratiques et expériences, et se lire aussi dans leurs objets scientifiques. Le problème n'est pas de nier la part cognitive du projet géographique, mais de réfléchir aux conséquences sur le savoir géographique de la méthode fondée sur le modèle du care, compte tenu du type d'expériences auquel elles donnent lieu, de retrouver le savoir géographique au bout d'autres motifs de la méthode que cognitifs et des expériences transférentielles qu'ils installent. C'est un enjeu épistémologique parce qu'il contient en lui la question de l'esthétique des savoirs géographiques.

\section{Avec la théorie transitionnelle : une esthétique haptique au fondement du savoir géographique}

« Si je devais résumer la situation des pays occidentaux et peut-être de l'humanité entière en ce XXème siècle finissant, je porterai l'accent sur la nécessité de mettre des limites (...). (...) Pour m'en tenir à un domaine qui ne me touche plus seulement comme simple citoyen mais dont je fais l'expérience professionnelle quasi quotidienne, le changement dans la nature de la souffrance des patients qui demandent une psychanalyse est significatif depuis trente ans que j'exerce (...). (...) Actuellement plus de la moitié de la clientèle psychanalytique est constituée par ce qu'on appelle des états limites et/ou des personnalités narcissiques (...). (...) Ainsi, une tâche urgente, psychologiquement et socialement, me semble-t-elle être celle de reconstruire les limites, de se redonner des frontières, de se reconnaître des territoires habitables et vivables - limites, frontières à la fois qui instituent des différences et qui permettent des échanges entre les régions (du psychisme, du savoir, de la société, de l'humanité) ainsi délimitées. » (Anzieu, 1995: 28-30).

Nous disposons avec le corpus de la psychanalyse transitionnelle (notamment, Winnicott, 2005 [1971]; Anzieu, 1995 ; Roussillon, 1999 ; Tisseron, 1995) des outils qu'il nous faut pour appliquer le principe de symétrie à l'expérience relationnelle de terrain du/de la géographe qualitativiste et pour penser un nouveau, un autre, régime de connaissance spatiale/géographique et ses objets, ainsi que ses rapports avec les régimes précédemment identifiés. J'ai élaboré cette proposition théorique dans plusieurs textes (par ex. Volvey, 2004, 2012a, 2012b), mais c'est ici sur la question du corps que je centrerai mon argumentation. Cette théorie relationnelle (nourrisson/environnement maternant ou 
analysant/thérapeute) de la créativité construite sur la pulsion d'attachement, le sens du toucher et la fonction symboligène de la peau, met au centre de sa théorie de la subjectivation et de sa théorie de la clinique le rapport entre corporalité et spatialité. Un rapport que l'expression «faire avec l'espace » construite et mobilisée par la géographie contemporaine permet d'élaborer avant de l'appliquer à l'intelligibilité d'une esthétique des savoirs géographiques issus d'une expérience relationnelle de terrain réglée par des motifs subjectifs inconscients et fonctionnant sur le principe d'un transfert de situation. Un rapport qui appelle à re-saisir le corps du/de la chercheure au terrain, non pas d'abord dans sa dimension sociale (nommée/nommable), mais à l'endroit de sa réalité vivante.

En résumé, pour la transitionnalité la construction narcissique-identitaire trouve à s'étayer doublement (construction en ellipse) sur l'environnement maternant puis, autoréflexivement grâce au sens du toucher sur la peau du nourrisson, dans une dynamique pulsionnelle (l'attachement) qui règle l'évolution spatiale de la situation de soin (l'échopraxie) tactilement éprouvée par la peau touchée/touchant, et qui règle sa psychisation en images du corps (« Moi-peau » chez Anzieu, par exemple) dont les fonctions sont dérivées des fonctions biologiques de la peau (fonctions de contenance, d'individuation, de communication, etc.). Les données pré-verbales, haptiques (toucher et empathie), transposées au niveau psychique, adoptent un mode figuratif (« images-sensations »), c'est-à-dire un mode spatial d'élaboration psychique - par opposition au mode séquentiel du langage verbal. L'ensemble du processus de figuration constitutif du Moi-peau s'apparente à un processus de lever topographique - sorte de somatopographie -, et à une conversion de type cartographique de ces données haptiques - sorte de somacartographie (ou mapping). Les types de figuration du moi qui en découlent sont le territoire et la discontinuité (frontière et interface). Plusieurs auteurs ont développé les conséquences cliniques de la théorie transitionnelle, abandonnant le modèle freudien de la cure fondé sur le rêve qui a contribué à désactiver le corps (analysant/analyste) et a participé à la liaison exclusive du sort de la psychanalyse au langage (talking cure), pour un modèle fondé sur le care et mobilisant notamment les actes de figuration (dessin, modelage). Ils font du cadre analytique, le moyen pour symboliser et le dispositif à symboliser, avec quoi il est possible pour les personnalités narcissiquesidentitaires de «vivre créativement», afin que rejoue le non ou le mal advenu de soi (voir Roussillon, 2008). Car le cadre, c'est-à-dire la relation dynamique de corps coprésents interagissant entre eux et avec l'espace clinique, est réputé contenir symbolisée en lui la problématique relationnelle inconsciente du sujet. Il est ce avec quoi les situations cliniques deviennent transférentielles. Cette théorie du cadre est, selon ces auteurs, transposable à tout type de situation où la spatialité et la corporalité se trouve nouées par des agirs relationnels. Elle peut l'être notamment, aux situations de terrain associées au développement des méthodes qualitatives (Volvey, 2012b).

L'analyse de cet ensemble théorique permet de fonder de manière cohérente la pensée d'une esthétique haptique en géographie à partir d'une analyse des éléments constitutifs de la méthode relationnelle (terrain et figuration) et d'en appréhender ses objets (territoire et discontinuité), tout en les liant doublement à la question contemporaine de l'identité subjective et au «tournant spatial» des sciences contemporaines. Elle permet de retrouver la dimension cognitive de la discipline scientifique au-delà des motifs identitaires inconscients qui animent l'activité de recherche. Il permet aussi de penser les autres régimes (scopiques et linguistiques) non pas dans l'opposition avec celui-ci, mais dans une concaténation 
dynamique supportée par à un continuum d'expériences réglé par la question de la gestion du problème de la distance par le sujet-cherchant via son engagement dans ses pratiques scientifiques. Ainsi, depuis les expériences haptiques jusqu'aux expériences discursives en passant par les expériences visuelles, c'est bien la limite qui est éprouvée comme contenant et interface d'abord à même la surface du corps (sur la peau), avant d'être retournée et projetée au-devant de soi dans l'horizon, dégageant alors un territoire partageable dans l'interdiscursivité. 